\title{
Blood flow changes in pelvic vessels associated with the application of an abdominal compression belt in healthy postpartum women
}

\author{
T Dias', M Patabendige ${ }^{2}$, RP Herath', TI Garvik ${ }^{3}$, F Liland ${ }^{3}$, S Arulkumaran ${ }^{4}$
}

\begin{abstract}
Introduction Postpartum haemorrhage (PPH) accounts for a high proportion of maternal mortality and morbidity throughout the world. A uterine compression belt which has been developed recently represents a very low tech, low cost solution in managing postpartum haemorrhage.

Objectives To evaluate the blood flow changes in pelvic vessels following application of the postpartum haemorrhage compression belt (Laerdal Global Health, Stavanger, Norway).

Methods The sample included healthy postpartum women within 6 hours of vaginal delivery. The study was performed at Teaching Hospital, Ragama, Sri Lanka. PPH compression belt was applied on the lower abdomen in a supine position with a slight lateral tilt. Patient's pulse, blood pressure and Doppler indices (RI, PI and PFV) of the uterine, internal iliac and femoral arteries were measured using transabdominal Doppler ultrasonography. Lower limb oxygen saturation was also measured. Measurements were obtained by connecting the subjects to a multimonitor throughout the study period of 20 minutes. Median $\mathrm{RI}, \mathrm{PI}$ and PFV was calculated and comparisons were made between the baseline and after belt application at 10 and 20 minutes.
\end{abstract}

Results A total of 20 healthy women were included and the mean time from delivery to study inclusion was 2.5 (range 0.5-5.0) hours. There were no adverse outcomes or altered vital signs noted among participants. Overall there were no significant changes in the internal iliac, uterine and femoral artery blood flow after application of the compression belt.

Conclusions There were no significant changes in the internal iliac, uterine and femoral artery blood flow after application of the compression belt. This preliminary study only shows that the application of the PPH compression belt has no apparent adverse changes in the iliac, uterine and femoral artery blood flow in postpartum mothers.

\section{Introduction}

Postpartum haemorrhage $(\mathrm{PPH})$ is responsible for a high proportion of maternal mortality worldwide. In resource limited countries, obstetric haemorrhage remains one of the major killers of mothers along with sepsis and hypertension. More than $30 \%$ of the estimated 500,000 maternal deaths that occur globally each year are attributed to postpartum haemorrhage [1]. Long delays in reaching emergency obstetric care are the main reason for many maternal deaths. Sri Lanka has been exceptional in achieving World Health Organization (WHO) millennium development goals in maternal care [2]. The latest maternal mortality ratio (MMR) for Sri Lanka is 37.7 per 100000 live births in 2013 and MMR remained low compared to other South Asian countries [2,3]. Despite the relative low figures, obstetric haemorrhage is an important cause of maternal deaths in Sri Lanka [4].

Evidence shows that a systematic approach to management of postpartum haemorrhage improves maternal outcome [5]. While the mother is resuscitated with fluids and blood, bimanual compression of the uterus is often undertaken to achieve heamostasis as the first-line of care along with administration of oxytocics. Compression of aorta may also be used to gain temporary control of bleeding until definitive treatment is commenced. External aortic compression involves the two hands lifting the uterine fundus up out of the pelvis, the lower hand then pushes upwards and backwards on the lower uterine segment while the other hand pushes the fundus firmly down on to the aorta. An alternative technique is to use the fist applied directly in the midline just above the umbilicus and the uterus, with the heel of the hand pressing down on the aorta (Fig. 1).

Both these techniques of bimanual compression of uterus and aortic pressure are based on the principle of mechanical compression or tamponade of uterine blood sinuses or aorta to slow down the pelvic circulation and control bleeding.

Ceylon Medical Journal 2017; 62: 228-232

DOI: http://doi.org/10.4038/cmj.v62i4.8572

1Department of Obstetrics and Gynaecology, Faculty of Medicine, University of Kelaniya, Sri Lanka.2University Obstetrics Unit, North Colombo Teaching Hospital, Ragama, Sri Lanka, 3Laerdal Global Health, Stavanger, Norway, 4Department of Obstetrics and Gynaecology, St George's Hospital and University of London, London, United Kingdom.

Correspondence: TD, e-mail: <thiran_dias@yahoo.com>. Received 06 July 2017 and revised version accepted 26 September 2017

This is an open-access article distributed under the terms of the Creative Commons Attribution License, which permits unrestricted use, distribution, and reproduction in any medium, provided the original author and source are credited. 
A PPH belt has been introduced recently in order to achieve same tamponade effect of bimanual uterine compression and aortic compression. The belt is worn on the abdomen, it is simple to use, can be applied rapidly and may help reduce the bleeding and avoid the need for a hysterectomy. It can be used while resuscitation, transfer or more definitive actions are being undertaken. This is more important in resource constrained settings where delay in definitive care may cost lives. In order to conduct definitive clinical trials to demonstrate the effectiveness of PPH compression belt in reducing blood loss and avoiding adverse outcomes, preliminary studies of its effect on pelvic circulation in healthy postpartum women are essential. The aim of this study was to evaluate the blood flow changes in pelvic vessels with the PPH compression belt (Laerdal Global Health, Stavanger, Norway), (Figure 2).

\section{Methods}

This was a prospective interventional study performed at North Colombo Teaching Hospital, Ragama, Sri Lanka between January 2017 and April 2017. All healthy, female, postpartum volunteers who provided written informed consent were recruited within 6 hours of vaginal delivery. Women with any underlying health problems, blood loss more than $500 \mathrm{ml}$ postpartum, delivered more than 6 hours ago, non-singleton pregnancy, preeclampsia, pulmonary or cardiac problems, high risk of thromboembolism, new born in poor condition, and less than 18 years of age were excluded from the study.

After obtaining consent, the PPH compression belt (Laerdal Global Health, Stavanger, Norway) was applied on the lower abdomen of the mother in a supine position with a slight lateral tilt. The compression belt consists of a rigid tray and with two belts placed around the patient's back, which can easily be hand-tightened to allow the rigid tray to apply pressure onto the abdomen. The rigid tray has two segments; a hard wedge which fits above the pubic symphysis to compress the lower uterine segment and steady the uterus and a hard plateau to provide pressure to the abdomen directly over the uterus (Figures 2, 3, 4). This devise is $240 \mathrm{~mm}$ wide, $180 \mathrm{~mm}$ long and $120 \mathrm{~mm}$ thick. The device was a prototype that was specially made for use in clinical studies. As a prototype, the price of one unit cannot be estimated. In a hypothetical case where the device is developed for cost-effective volume production, the unit price would likely be in the range of 30-40 USD.

The objective of the study was to estimate the change in blood flow to the pelvis with application of the compression belt over the lower abdomen. The belt was tightened sufficiently to exert a reasonable amount of pressure on the uterus without causing undue discomfort to the woman.

Patient's pulse and brachial artery blood pressure were measured using a sphygmomanometer. Resistance index, pulsatility index and peak systolic velocity (RI, PI and PFV) of the uterine, internal iliac and femoral arteries were measured using transabdominal Doppler ultrasonography. Lower limb oxygen saturation was also measured. Women were connected to a multi-monitor
Table 1. Demographic and clinical characteristics of the study participants

\begin{tabular}{ll}
\hline & Mean (range) \\
\hline Age (years) & $26(19-33)$ \\
Parity & $1.6(1-4)$ \\
Body mass index $(\mathrm{kg} / \mathrm{m} 2)$ & $25.3(19.1-39.4)$ \\
$\begin{array}{l}\text { Gestational age at time of } \\
\text { delivery (weeks) }\end{array}$ & $39.1(37-40)$ \\
$\begin{array}{l}\text { Symphysio-fundal height } \\
\text { (cm) }\end{array}$ & $18.8(10-24)$ \\
$\begin{array}{l}\text { Abdominal circumference at } \\
\text { uterine fundus (cm) }\end{array}$ & $81.3(66.0-96.0)$ \\
$\begin{array}{l}\text { Abdominal circumference at } \\
\text { pubic symphysis (cm) }\end{array}$ & $87.0(73.0-101.0)$ \\
$\begin{array}{l}\text { Distance from umbilicus to } \\
\text { uterine fundus (cm) }\end{array}$ & $1.4(0-3)$ \\
\hline
\end{tabular}

throughout the study period of 20 minutes for these measurements. Median RI, PI and PFV was calculated and comparisons were made between the baseline and after belt application at 10 and 20 minutes. A semi-inflated mercury sphygmomanometer cuff was placed on the inner aspect of the compression belt to note the pressures achieved during the study. The pressure within the belt was kept between 20-30 mmHg. Pressure was increased up to a maximum of $30 \mathrm{mmHg}$ unless patient felt any discomfort.

The duration for which the belts were on was recorded in all women. Women were asked to report any side effects such as pain, weakness, numbness, loss of sensation or discolouration of lower extremities and any other ill effects they experience over the next 24 hours. The researcher checked the nail bed perfusion time and any discoloration of the lower extremities and oxygen saturation of the big toe during the procedure. If any of these symptoms or signs developed the belt was released immediately.

The study was approved by the Ethics Committee of the Faculty of Medicine, University of Kelaniya. Ethical and scientific approval was also obtained from the St. George's University of London which is the main collaborator of this study. Data was entered into a Microsoft Excel spreadsheet. Statistical analysis was done using SPSS software. Wilcoxon Signed Rank test was used to see any significant difference between measurements. $\mathrm{P}$ values $<0.05$ was considered as statistically significant.

\section{Results}

The sample included a total of 20 healthy postpartum women. Basic characteristics of the study population are given in the table 1. Mean pressure within the belt was $26.5(\mathrm{SD}=1.76) \mathrm{mmHg}$ and the range was $24.0-30.0$ $\mathrm{mmHg}$. There was a statistically significant correlation between pressure within the belt and BMI (Spearman's 
Table 2. Blood flow changes in pelvic vessels before, during and after the release of the PPH belt

\begin{tabular}{llllllll}
\hline Vessel & $\begin{array}{l}\text { Time } \\
\text { after belt } \\
\text { application }\end{array}$ & $\begin{array}{l}\text { Resistance } \\
\text { Index (SD) }\end{array}$ & $\begin{array}{l}\text { P Value } \\
\text { (compared } \\
\text { to baseline) }\end{array}$ & $\begin{array}{l}\text { Pulsatility } \\
\text { Index (SD) }\end{array}$ & $\begin{array}{l}\text { P Value } \\
\text { (compared } \\
\text { to baseline) }\end{array}$ & $\begin{array}{l}\text { Peak Flow } \\
\text { Velocity } \\
\text { (SD) }\end{array}$ & $\begin{array}{l}\text { P Value } \\
\text { compared } \\
\text { to baseline) }\end{array}$ \\
\hline $\begin{array}{l}\text { Internal Iliac } \\
\text { artery }\end{array}$ & Baseline & $0.9(0.13)$ & - & $1.8(0.85)$ & - & $78.0(28.2)$ & \\
& 10 minutes & $0.9(0.12)$ & 0.6 & $1.8(0.75)$ & 0.69 & $70.8(27.9)$ & 0.3 \\
& 20 minutes & $0.84(0.16)$ & 0.12 & $1.9(0.66)$ & 0.22 & $74.1(28.1)$ & 0.27 \\
& 10 minutes & $0.91(0.09)$ & & $2.08(0.75)$ & & $70.0(20.2)$ & \\
& $\begin{array}{l}\text { after release } \\
\text { of belt }\end{array}$ & & & & & & \\
\hline $\begin{array}{l}\text { Uterine } \\
\text { artery }\end{array}$ & Baseline & $0.79(0.19)$ & - & $1.5(0.69)$ & - & $75.9(16.4)$ & - \\
& 10 minutes & $0.72(0.17)$ & 0.7 & $1.3(0.71)$ & 0.7 & $94.9(21.4)$ & P $<0.05$ \\
& 20 minutes & $0.76(0.17)$ & 0.7 & $1.5(0.62)$ & 0.9 & $85.6(26.9)$ & 0.2 \\
& 10 minutes & $0.63(0.18)$ & & $1.03(0.71)$ & & $83.8(21.9)$ & \\
& after release \\
of belt & & & & & & \\
\hline $\begin{array}{l}\text { Femoral } \\
\text { artery }\end{array}$ & Baseline & $0.9(0.07)$ & - & $1.7(0.13)$ & - & $66.9(18.0)$ & - \\
& 10 minutes & $0.9(0.13)$ & 0.18 & $1.8(0.42)$ & 0.56 & $68.1(18.4)$ & 0.2 \\
& 20 minutes & $0.9(0.15)$ & 0.42 & $1.5(0.46)$ & 0.18 & $64.1(13.3)$ & 0.1 \\
& 10 minutes & $0.9(0.07)$ & & $1.55(0.56)$ & & $59.3(12.3)$ & \\
& $\begin{array}{l}\text { after release } \\
\text { of belt }\end{array}$ & & & & & & \\
\hline
\end{tabular}

rho 0.74, P value < 0.01). Details of RI, PI and PFV at baseline, 10 minutes after belt application and 20 minutes after belt application are given in table 2. Application of the compression belt did not show any significant changes in pulse rate, blood pressure or limb oxygen saturation. PPH compression belt neither appeared to cause any discomfort nor side effects after application.

\section{Discussion}

PPH compression belt represents a very low tech, low cost solution to postpartum haemorrhage due to uterine atony. The application of this apparatus does not require extensive training of health care personnel and would be very useful in under-resourced setting, where postpartum haemorrhage continues to be a serious problem. This device would also be useful in non-hospital situations where there is a limited number of staff.

Similar devices have been introduced recently to achieve a tamponade effect. An external aortic compression device (EACD) which is a hand-made spring device held in place by a leather belt, was used to compress the aorta. EACD has been shown to reduced time to cessation of uterine bleeding significantly in one study; however, additional research is necessary to determine the effectiveness of this device [6]. "Sumocompression" after vaginal delivery in patients with severe postpartum haemorrhage has been reported [7]. The uterus is compressed from below by tight packing of the vagina with $3-5$ sterile gauze pads. A bandage is fixed like a very tight belt preventing the uterus from rising and a roll of pads compresses the uterus from the fundus. Counter-pressure is achieved by a second bandage from the anterior to the posterior part of the belt pressing the pads inside the vagina firmly against the uterus. The hip areas are padded to reduce skin trauma.

Abdominal compression with sand bags which was used several decades ago seems to have a similar effect. The use of non-pneumatic anti-shock garments (NASG) is recommended as a temporizing measure until substantive care is available $[9,10,11]$. The NASG is a low-tech firstaid, re-usable lower-body compression garment which can be used to stabilise women with postpartum haemorrhage $[10,11]$. It can be used for postpartum haemorrhage of any aetiology, can be applied by individuals with minimal training, and does not affect the use of other postpartum haemorrhage management interventions [10,11]. Quasiexperimental studies at tertiary care facilities have shown significantly reduced measured blood loss, more rapid recovery from shock and decreased mortality $[10,11]$.

Our study demonstrates that the blood flow through femoral artery and limb oxygen saturation were not affected by application of the PPH compression belt. This indicates that the device is safe for use in postpartum haemorrhage. However, we were unsalable to show 


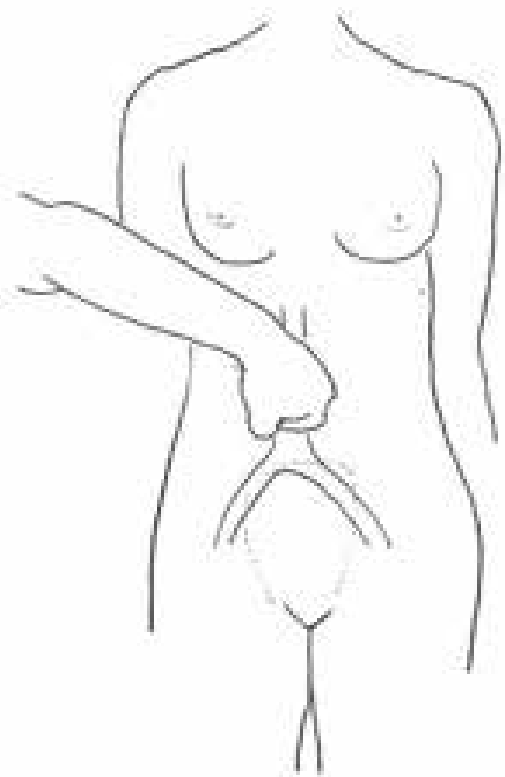

Figure 1. Aortic compression

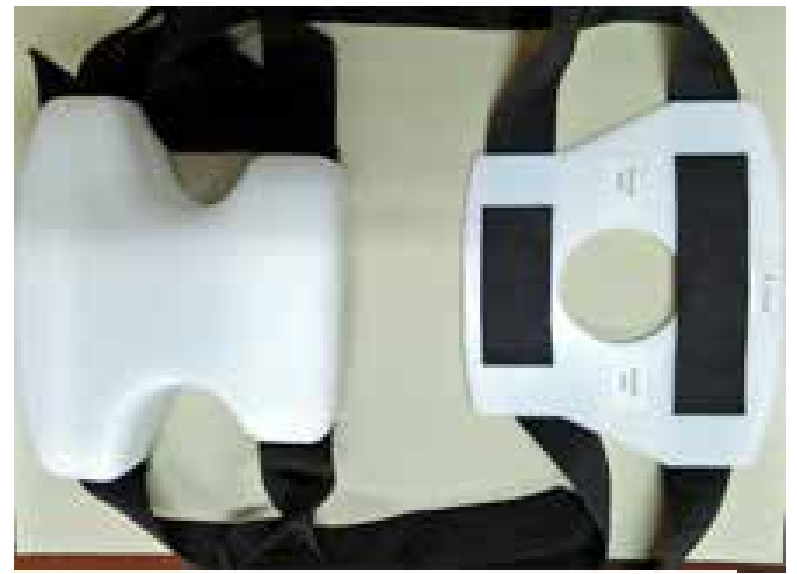

Figure 3. The PPH compression belt

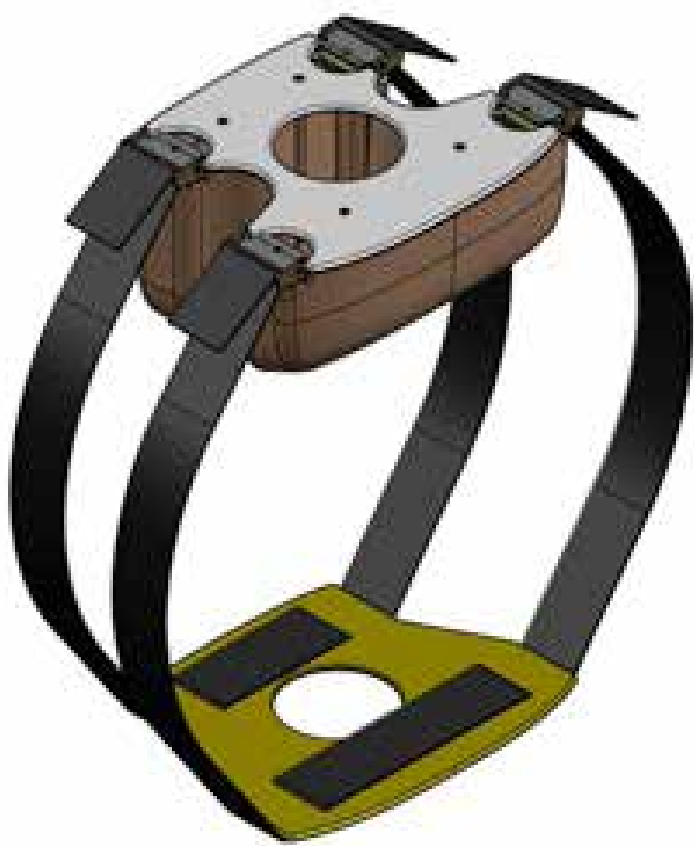

Figure 2. The PPH compression belt

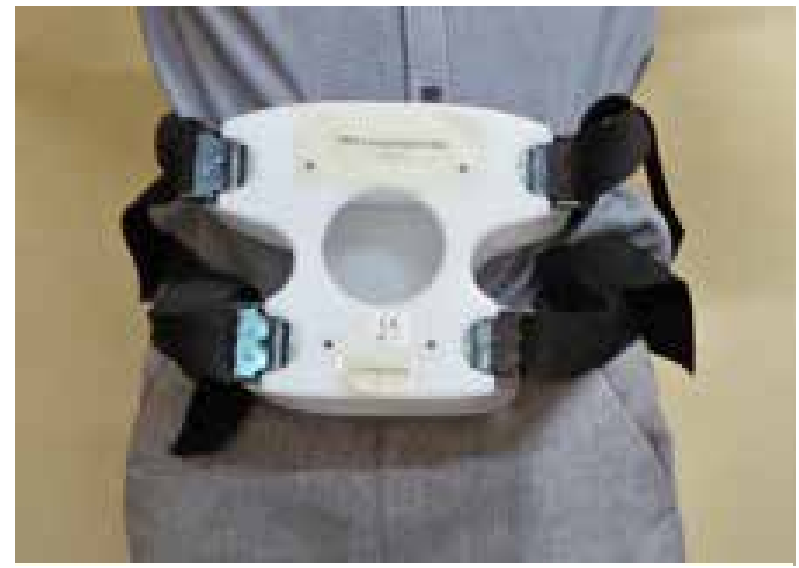

Figure 4. Application of the PPH compression belt on the lower abdomen (placing of the semi-inflated mercury sphygmomanometer cuff not shown) 
significant changes in Doppler indices of internal and uterine arteries when comparing baseline data and those after application of the belt. A pilot study had been done using another compression belt developed by the same manufactures in 2015. This study which measured only RI values in pelvic vessels reported no significant effect [8]. The device used in our study is a modification of the device used in the study in 2015 [8].

Further modification of the device and larger studies using 3D power Doppler and intrauterine pressure monitoring are likely to provide better understanding of the physiological impact of the PPH compression belt in women with postpartum haemorrhage.

This preliminary study only shows that there are apparently no adverse effects on the postpartum mother. The study did not look at its effectiveness in the management of postpartum haemorrhage. If it is shown to be effective and safe it would be useful is any settings for the management of an atonic postpartum haemorrhage, but especially in an under-resourced settings. Advantages of this device are that it would be a low tech, low-cost device, which is user friendly, and its application would not require extensive training of health care personnel or a high degree of skill to operate correctly. This device would also be useful for non-hospital situations where there are frequently a limited number of staff. Also it can be easily manufactured and enables hands free compression in nonhospital and transport environments. In high-resourced countries, this device could be used when a bleeding patient needs emergency transport to a different location. It may be useful even in cases of trauma or coagulopathy as an interim measure.

\section{Acknowledgment}

We would like to thank all women who participated in the study.

\section{Conflicts of interest}

Authors declare that they have no conflicts of interest. The study did not receive any direct funding.

\section{References}

1. Khan KS, Wojdyla D, Say L, Gulmezoglu AM, Van Look PF. WHO analysis of causes of maternal death: a systematic review. Lancet 2006; 367:1066-74.
2. Senanayake H, Goonewardene M, Ranathunga A, Hattotuwa R, Amarasekara S, Amarasinghe I. Achieving Millennium Development Goals 4 and 5 in Sri Lanka. BJOG- An Int J Obstet Gynaecol 2011; 118: 78-87.

3. Lozano R, Wang H, Foreman KJ et.al. Progress towards Millennium Development Goals 4 and 5 on maternal and child mortality: an updated systematic analysis. Lancet 2011; 24 378(9797):1139-65.

4. Senanayake H, Dias T, Jayawardena A. Maternal mortality and morbidity: epidemiology of intensive care admissions in pregnancy. Best Pract Res Clin Obstet Gynaecol 2013; 27(6):811-20.

5. Skupski DW, Lowenwirt IP, Weinbaum FI, Brodsky D, Danek M, Eglinton GS. Improving hospital systems for the care of women with major obstetric haemorrhage. Obstet Gynaecol 2006; 107(5): $977-$ 83.

6. Soltan MH, Sadek RR. Experience managing postpartum hemorrhage at Minia University Maternity Hospital, Egypt: no mortality using external aortic compression. J Obstet Gynaecol Res 2011; 37(11):1557-63.

7. Spätling L, Schneider H. ["Sumo-compression" Stops Post Partum Haemorrhage]. Z Geburtshilfe Neonatol 2014; 218(5):223-5.

8. Sinai Talaulikar V, Khalil A et.al. A pilot study of the impact of a novel compression belt on pelvic blood flow in healthy postpartum women. Int J Gynaecol Obstet 2015; 131(2):208.

9. World Health Organization. WHO recommendations for the prevention and treatment of postpartum hemorrhage. (2012). Available at: http://apps.who. int/iris/bitstream/10665/75411/1/9789241548502 eng.pdf. Accessed on 26 Aug 2017.

10. Miller S, Fathalla MM, Ojengbede OA et al. Obstetric hemorrhage and shock management: using the low technology Non-pneumatic Anti-Shock Garment in Nigerian and Egyptian tertiary care facilities. BMC Pregnancy Childbirth 2010;10: 64

11. Miller S, Turan JM, Dau K et al.Use of the nonpneumatic anti-shock garment (NASG) to reduce blood loss and time to recovery from shock for women with obstetric haemorrhage in Egypt. Global Public Health 2007; 2(2): 110-124 